\title{
The influence of the band structure of epitaxial graphene on SiC on the transistor characteristics
}

Gong Gu

Sarnoff Corporation

201 Washington Rd, Princeton, NJ 08543-5300, USA

Luxmi, P. J. Fisher, N. Srivastava, R. M. Feenstra

Department of Physics, Carnegie Mellon University

Pittsburgh, PA 15213, USA

\begin{abstract}
We fabricated high-mobility field-effect transistors based on epitaxial graphene synthesized by vacuum graphitization of both the Si- and C-faces of SiC. Roomtemperature field-effect mobilities $>4,000 \mathrm{~cm}^{2} / \mathrm{Vs}$ for both electrons and holes were achieved, although with wide distributions. By using a high-k gate dielectric, we were able to measure transistor characteristics in a wide carrier density range, where the mobility is seen to decrease as the carrier density increases. We formulate a simple semiclassical model of electrical transport in graphene, and explain the sub-linear dependence of conductivity on carrier density from the view point of few-layer graphene energy band structure. Our analysis reveals important differences between few-layer graphene energy dispersion on the $\mathrm{SiC} \mathrm{Si-} \mathrm{and} \mathrm{C-faces,} \mathrm{providing} \mathrm{the} \mathrm{first} \mathrm{evidence} \mathrm{based}$ on electrical device characteristics for the theoretically proposed energy dispersion difference between graphene synthesized on these two faces of SiC.
\end{abstract}

\section{Introduction}

Graphene has attracted tremendous research interest, due to both its scientific significance and technological potentials [1], with the latter especially in ultrafast electronics. Field-effect transistors (FETs) have been demonstrated with roomtemperature mobilities up to $15,000 \mathrm{~cm}^{2} / \mathrm{Vs}$ using few layer graphene (FLG) exfoliated from graphite [2]. It is desirable for device manufacturing and integration, however, to synthesize graphene on a solid state substrate instead of exfoliation and placing. To this end, graphitization of $\mathrm{SiC}$ represents a promising approach towards epitaxial synthesis of graphene [3, 4]. Transistors using epitaxially synthesized graphene on SiC have been demonstrated [5, 6, 7].

In this work, we synthesized graphene on both the (0001) and (0001) surfaces (referred to respectively as the $\mathrm{Si}$ - and $\mathrm{C}$-faces hereafter) of $4 \mathrm{H} \mathrm{SiC}$, and fabricated graphene FETs using a high-k dielectric $\mathrm{HfO}_{2}$ as the gate dielectric. Room-temperature field-effect mobilities $>4,000 \mathrm{~cm}^{2} / \mathrm{Vs}$ were achieved. The use of the high-k dielectric enabled us to measure transfer characteristics in a wide carrier density range. We can therefore analyze the $I_{D^{-}} V_{G}$ data and explain the bending of the $I_{D^{-}} V_{G}$ curves in terms of the band structure of FLG. The physical picture we present is consistent with data in early work [7, 8]. Our analysis revealed important differences between few-layer graphene energy dispersion on the $\mathrm{SiC} \mathrm{Si-} \mathrm{and} \mathrm{C-faces,} \mathrm{providing} \mathrm{the} \mathrm{first} \mathrm{evidence} \mathrm{based}$ on electrical device characteristics for the theoretically proposed energy dispersion difference between graphene synthesized on the two faces of SiC. 
Section 2 describes device fabrication and characterization. Section 3 and 4 present data analysis for devices using graphene synthesized on the C- and Si-faces, respectively. Section 5 draws conclusions as well as discusses our physical picture as related to data in early work. Mathematical details are in the Appendix.

\section{Experiment}

Our epitaxial synthesis and thickness estimate of graphene on the Si-face have been described elsewhere [9]. The average thicknesses of both Si-face samples were estimated to be 2 ML by Auger electron spectroscopy (AES) (this thickness does not include the C-rich "buffer layer" at the SiC/graphene interface [9]). The synthesis on the C-face starts from a hydrogen etched substrate, and graphene formation is observed over the temperature range $1200-1400^{\circ} \mathrm{C}$. The low energy electron diffraction (LEED) pattern of the Si-face graphene reveals a six-fold pattern, the same as described in the literature [3, 4]. The pattern of the C-face graphene is more complex, revealing a six-fold pattern together with additional spots at $30^{\circ} \pm \varphi$ with angles $\varphi$ ranging from 6 to $13^{\circ}$. This pattern is similar to that observed by Hass et al [4] (although their angle $\varphi$ was $2.2^{\circ}$ ), and following those authors we interpret it as indicative of rotational disorder in the C-face graphene layers. Thickness estimate by AES was not reliable for thicker films on the Cface, and for that reason we use heights as measured by atomic force microscopy (AFM) as further discussed below.

Transistors with the structure shown in Fig. 1(a) were fabricated at Lincoln Labs in a process described elsewhere [7], using two Si- and two C-face graphene samples as listed Table I, each $1 \mathrm{~cm} \times 1 \mathrm{~cm}$ in size. The $\mathrm{HfO}_{2}$ gate dielectric is $40 \mathrm{~nm}$ thick with a dielectric constant $\varepsilon_{r}=23$, resulting in a unit area gate capacitance $C_{i}=509 \mathrm{nF} / \mathrm{cm}^{2}$.

The patterned graphene is totally covered by the gate/dielectric stack, the source/drain (S/D) metals, or their overlap. Edges of the graphene islands translate to edges in the top surface. The thickness of the graphene films on the C-face was estimated by measuring by AFM the average height of the covered graphene islands in completed devices. This measurement avoids the uncertainty caused by different interaction of the AFM tip with different materials [2], but assumes that the $\mathrm{HfO}_{2}$ or $\mathrm{S} / \mathrm{D}$ metal films have same thicknesses on graphene and bare $\mathrm{SiC}$. To verify the AFM result, transmission electron microscopy (TEM) was performed on one device on sample 20 (C-face). The high resolution images (to be published) reveals $\sim 27$ graphene MLs with a total thickness of $\sim 9 \mathrm{~nm}$, as well as a 3 to $4 \mathrm{~nm}$ thick unknown layer between epitaxial graphene and $\mathrm{HfO}_{2}$. This unknown layer is tentatively attributed to an organic residue. The TEM result indicates that the AFM-measured height is the actual graphene thickness plus 3 to $4 \mathrm{~nm}$ of the unknown layer, consistent with the 12 to $13 \mathrm{~nm}$ AFM-measured height on the two C-face samples.

Figure 1(b) shows the channel conductivity $\sigma$ of many devices on sample 20 (Cface), measured at room temperature with a drain voltage $V_{D}=0.1 \mathrm{~V}$, versus the gate voltage $V_{G}$. Here, we name the grounded electrode $\mathrm{S}$, and the biased contact $\mathrm{D}$, although this is not perfectly appropriate for the ambipolar FETs [7]. For a low $V_{D}=0.1 \mathrm{~V}$, however, the difference between the electron and the hole sources is simply ignored. Similar to [7], large non-uniformity was observed. Field-effect mobilities, $\mu$, were calculated from the largest slopes of the transfer curves above and below the minimum conductance voltage $V_{m}$ for electrons and holes, respectively. Figure 1(c) shows $\sigma$ - $V_{G}$ 
curves for two representative devices on sample 29 (Si-face), featuring wide linear portions of the curves near $V_{m}$ to be discussed later; the non-uniformity among devices for this face is also large.

\section{Analysis of $\mathbf{C}$-face graphene FETs}

Figure 1(b) shows that the $\sigma-V_{G}$ curves bend downward at high gate biases, i.e. the mobility decreases with increasing $\left|V_{G}-V_{m}\right|$. To show this effect more clearly, the $\sigma-V_{G}$ curve of one representative FET on this sample is shown in Fig. 2.

To explain this behavior, we start from monolayer graphene, with a linear energy dispersion $E=\hbar v_{F} k$, where $k$ is the wavevector and $v_{F} \approx 10^{8} \mathrm{~cm} / \mathrm{s}$ the Fermi velocity. For simplicity, we assume that carrier scattering can be characterized by a scattering time $\tau$ without considering the microscopic scattering mechanisms. Under an applied electric field $F$, the carrier distribution in the momentum space is shifted by $\hbar \Delta k=q F \tau$, where $q$ is the electron charge. While the velocity of each carrier is still $v_{F}$, a net drift velocity $v_{d}$ results. A simple expression can be found by averaging the velocity projection along the field direction over all carriers for zero absolute temperature (see Appendix):

$$
v_{d}=v_{F} \frac{\Delta k}{k_{F}}=v_{F} \frac{q F \tau}{\hbar k_{F}}=v_{F}^{2} \frac{q F \tau}{E_{F}}
$$

For temperature $T=0 \mathrm{~K}$, we also have

$$
E_{F}=\hbar v_{F} k_{F}=\hbar v_{F} \sqrt{\pi n} \text {, }
$$

where $n$ is the carrier density, considering the spin degeneracy of 2 and the valley degeneracy of $2[10,11]$. Therefore, the conductivity

$$
\sigma=q n\left(\frac{q v_{F}{ }^{2}}{E_{F}} \tau\right)=\frac{q^{2} v_{F}}{\hbar \sqrt{\pi}} \cdot \sqrt{n} \cdot \tau .
$$

The general case of $T>0 \mathrm{~K}$ is discussed in the Appendix, where Eq. (3) is corrected by a factor that depends only on the ratio $E_{F} /\left(k_{B} T\right)$. For $n>\sim 4 \times 10^{12} \mathrm{~cm}^{-2}$, the temperature correction is negligible even at room temperature.

In a graphene FET, the minimum conductivity gate voltage $V_{m}$ is usually not $0 \mathrm{~V}$, due to unintentional doping, and hence the carrier density $n=C_{i}\left(V_{G}-V_{m}\right) / q$. Therefore, Eq. (3) means that $\sigma \propto \sqrt{\left|V_{G}-V_{m}\right|}$ at sufficiently high carrier densities and low temperatures, assuming a constant $\tau$. This is consistent with the transfer characteristics of the back-gated single layer graphene FET demonstrated by Lemme et al (Fig. 2 of Ref. [8]). Indeed, the Appendix shows that our semiclassical model is consistent with that transfer curve all over the gate bias range when finite temperature is considered (see Fig. A2).

Back to our device in Fig. 2, we first determine the minimum conductance voltage $V_{m}=0.69 \mathrm{~V}$ by linearly extrapolating the several data points near the minimum conductivity on both sides. We then fit the data to $\sigma=K \sqrt{\left|V_{G}-V_{m}\right|}+\sigma_{0}$ for $1.6 \mathrm{~V} \geq \mid V_{G}$ I $\geq 0.8 \mathrm{~V}$ for the hole conduction part of the curve. The offset parameter $\sigma_{0}$ was introduced for the following reason: The minimum conductivity of an epitaxial graphene FET on SiC is more than can be explained by thermally excited carriers. Such epitaxial graphene FETs thereby exhibit significantly lower on-off ratio [6, 7] than their exfoliated graphene counterparts $[2,8]$. Since the mechanism of the minimum conductivity is 
unclear, we introduce $\sigma_{0}$ as a fitting parameter. To fit the hole conduction, $\sigma_{0}=2.71 \mathrm{mS}$, reasonably close to the measured minimum conductivity.

In the $V_{G}$ range where we obtained the above fit, the effect of finite temperature is negligible: for $V_{G}=-0.8 \mathrm{~V}$, the hole concentration is $4.75 \times 10^{12} \mathrm{~cm}^{-2}$, where the temperature correction factor $\sqrt{G_{T}} \cdot F_{T} \approx 0.99$ (see Fig. A3 in the Appendix). We then consider room temperature using Eq. (A9), and fit the hole conduction portion of the curve to

$\sigma=K^{\prime} \sqrt{n} \cdot \sqrt{G_{T}\left(\frac{\hbar v_{F} k_{F}}{k_{B} T}\right)} \cdot F_{T}\left(\frac{\hbar v_{F} k_{F}}{k_{B} T}\right)+\sigma_{0}$,

where $\sqrt{G_{T}} \cdot F_{T}$ was calculated numerically (see Fig. A3), and $\sigma_{0}=2.71 \mathrm{mS}$ had been obtained by fitting the the temperature insensitive bias range. The hole density $n$ was related to $V_{G}$ by $q n=C_{i}\left|V_{G}-V_{m}\right|$. This fit is plotted in Fig. 2. Interestingly, for $\left|V_{G}\right| \leq$ $1.6 \mathrm{~V}$, very good fitting was obtained, suggesting that the band structure of our C-face FLG is linear, as of the single layer graphene. This is seemingly contradictory to FLG and graphite band structures that comprise parabolic conduction band bottoms and valence band tops $[2,12,13]$.

In this regard it should be noted that our C-face FLG is misoriented as evidenced by LEED, as discussed in Section 2, consistent with other work [3, 4, 14]. It has been shown $[4,15,16]$ that the energy dispersion of misorented FLG is linear, similar to the massless fermions of the single layer graphene, due to the loss of A-B stacking [14]. Indeed, the retention of the graphene linear band structure is considered an advantage of the epitaxial FLG on the SiC C-face over the Si-face counterpart $[4,14]$.

For $V_{G}$ more negative than $-1.6 \mathrm{~V}$, the $\sigma-V_{G}$ curve of this device bends down faster than the fit, and no reasonable fit to $\sigma=K \sqrt{\left|V_{G}-V_{m}\right|}+\sigma_{0}$ can be found if this range is included. Some other FETs even exhibit a decrease in $\sigma$ at high $\left|V_{G}\right|$ as shown in Fig. 1(b). Similar fast bend-down or even decrease in $\sigma$ at high $\left|V_{G}\right|$ was observed in other work using epitaxial graphene on $\mathrm{SiC}$ and the same dielectric deposition process [7], while a back-gated FET without deposited dielectric using exfoliated graphene [8] exhibit transfer characteristics consistent with Eq. (4) at all gate biases. This discrepancy will be discussed in Sect. 5. Gate leakage is excluded as a cause; the measured gate current in always negligible compared to the drain current.

The same trend is seen for electron conduction, although there are not enough data points for fitting due to the fast bend-down at high $V_{G}$ and a positive $V_{m}=0.69 \mathrm{~V}$.

\section{Analysis of Si-face graphene FETs}

Figure 3 shows the $\sigma-V_{G}$ curve of a well-behaved Si-face graphene FET on sample 25. Similar as in Fig. 2, $V_{m}=0.75 \mathrm{~V}$ was determined by linearly extrapolating the curve near the minimum conductivity. We then fit the data to $\sigma=K \sqrt{\left|V_{G}-V_{m}\right|}+\sigma_{0}$ for $2 \mathrm{~V} \geq\left|V_{G}\right|$ $\geq 1 \mathrm{~V}$ for the hole conduction part of the curve. Here, $\sigma_{0}=2.51 \mathrm{mS}$, reasonably close to the measured minimum conductivity. Similar to the above analysis of C-face FLG FETs, we attempted to fit the data over the range $-2 \mathrm{~V} \leq V_{G} \leq V_{m}$ to Eq. (4), taking into account the finite temperature correction. As plotted in Fig. 3, good agreement is seen only down 
to $V_{G}=-0.6 \mathrm{~V}$. At lower overdrive voltages $\left|V_{G}-V_{m}\right|$, however, the $\sigma-V_{G}$ data is linear.

To explain the behavior shown in Fig. 3, we consider the band structures of A-B stacked FLG or bilayer graphene [12, 13, 17]. The conduction (valence) bands are quadratic close to the bottoms (tops), and become linear at larger momentums [12, 13, 17]. At low $\left|V_{G}-V_{m}\right|$, the conduction (valence) band is filled up (down) to a level within the quadratic energy dispersion, therefore the transfer curve exhibit a constant slope (mobility) as expected for a conventional semiconductor with parabolic bands. At sufficiently high $\left|V_{G}-V_{m}\right|$, the Fermi level is beyond the transition between quadratic and linear dispersions, therefore the transfer curve resembles that of a single layer graphene FET.

Again, since $V_{m}=+0.75 \mathrm{~V}$, there are not enough electron conduction data for reliable fitting, but the trend of the curve is qualitatively the same: linear close to $V_{m}$ and sub-linear at higher $V_{G}$.

\section{Discussion and Conclusions}

We have presented a simple Drude-like model of electrical transport in graphene FETs to explain the decreasing mobility with increasing gate overdrive voltage $\left|V_{G}-V_{m}\right|$. This model reasonably fits the transfer curves of well-behaved transistors in our work. It is also consistent with data from a FET based on exfoliated, single layer graphene in other work [8]. Most interestingly, our fits reveal that the energy dispersion of FLG on the SiC $\mathrm{C}$-face is linear, while that on the Si-face comprise a quadratic portion at lower energies. The difference between the FET characteristics on the two faces is pronounced for all well-behaved devices in this work, and even for some less well-behaved devices: For the Si-face graphene, the linear portion of the $\sigma-V_{G}$ curve near the minimum conductivity is $\sim 1 \mathrm{~V}$, and the transition between the linear and sub-linear dependences is relatively abrupt. Figure 1(c) shows these features with two additional devices; the two are representative of well-behaved and less-well behaved device. On the C-face, however, the apparent linear range of the $\sigma-V_{G}$ curve is only $\sim 0.4 \mathrm{~V}$. The difference between the FLG films on the two faces is attributed to different orientational orders in the two cases. The validity of our simple model hinges on the assumption of a scattering time independent of the carrier density. Various scattering mechanisms, such as short range scatterers [18, 19], ionized impurities [18, 19], graphene acoustic phonons [19], surface phonons in the gate dielectric [20], and types of disorder including vacancies [19] and ripples [21], have been studied for exfoliated graphene. The early work by Novoselov et al [2] showed linear transfer characteristics up to $n \sim 7 \times 10^{12} \mathrm{~cm}^{-2}$, probably due to the dominance of Coulomb scattering [18, 19], where $\tau \propto \sqrt{n}$ leads to $\sigma \propto n$ in Eq. (3). Others observed sublinear $\sigma-n$ [22] due to short range scattering [18, 19]. For surface phonons, $\sigma \propto E_{F} \propto \sqrt{n}$ for realistic graphene-dielectric distances [20], implying near constant $\tau$. For our epitaxial graphene FETs with moderate mobilities compared to exfoliated graphene $[2,22]$, we expect many mechanisms to coexist, among which the surface phonons and morphological disorder dominate, due to the use of the high-k dielectric [20] and the rough surface morphology (see AFM images in Refs. [9] and [23]), respectively. For the morphological disorder, it is reasonable to assume the mean free path $v_{F} \tau$ as determined by surface roughness and average graphene domain size, thus 
independent of the carrier density. Given the coexistence of many mechanisms and the possible dominance of the two with near constant $\tau$, our assumption of a constant $\tau$ seems reasonable for a range of carrier densities. This assumption should also be reasonable for the back-gated device of Ref. [8], given the moderate mobility comparable to ours, although the scattering mechanisms may be different. The poor fits of our model to the data at high overdrive voltages, however, suggests the failure of this assumption at high carrier densities, where mechanisms such as short range scattering $[18,19]$ becomes more important.

In summary, we have fabricated high-mobility FETs using epitaxial graphene synthesized by vacuum graphitizing the $\mathrm{Si}$ - and $\mathrm{C}$-faces of $\mathrm{SiC}$. Analysis of the electrical data reveals the important difference between the band structures of FLG formed on the C- and Si-faces, providing the first device electrical characteristics evidence for the difference in energy dispersions of FLG on the two faces of SiC.

\section{Appendix}

Each carrier is shifted in the momentum space by $\hbar \Delta k=q F \tau$ by an electric field $F$. The velocity projection to the direction of the field is $v_{x}=v_{F} \cos \theta$, where the angles $\theta$ and $\theta$, are related by $\tan \theta^{\prime}=k \sin \theta /(\Delta k+k \cos \theta)$ as shown in Fig. A1. Hence the drift velocity is

$$
v_{d}=\frac{\int_{0}^{\infty} d k \int_{0}^{2 \pi} d \theta\left[k \cos \theta^{\prime} f(k)\right]}{2 \pi \int_{0}^{\infty} d k[k f(k)]} v_{F},
$$

where $f(k)=1 /\left\{1+\exp \left[\hbar v_{F}\left(k-k_{F}\right) / k_{B} T\right]\right\}$ is the Fermi distribution, and $k_{B}$ is the Boltzmann constant.

At this point, to simplify the physical picture, we consider the $T=0 \mathrm{~K}$ case, where Eq. (A1) reduces to

$$
v_{d}=\frac{\int_{0}^{k_{F}} d k \int_{0}^{2 \pi} d \theta\left[k \cos \left(\tan ^{-1} \frac{k \sin \theta}{\Delta k+k \cos \theta}\right)\right]}{\pi k_{F}^{2}} v_{F}
$$

consideration of finite temperatures is deferred until we discuss temperature dependence. Equation (A2) is still too complicated to analytically evaluate. Notice in Fig. A1 that only the carriers in the shaded areas contribute to the drift velocity. For $\Delta k<<k_{F}$, the contribution of the shaded area is easily calculated:

$$
v_{d}=\frac{2 \int_{-\pi / 2}^{\pi / 2}\left(v_{F} \cos \theta\right) \cdot \Delta k \cdot\left(k_{F} d \theta \cos \theta\right)}{\pi k_{F}{ }^{2}} \text {. }
$$

Taking the integral, we simply get Eq. (1) for $\Delta k<<k_{F}$ and $T=0 \mathrm{~K}$.

Although Eq. (A1) is general, we have so far only given an analytical expression of $v_{d}$ for $T=0 \mathrm{~K}$. Here we consider the general case of finite temperatures, once again for $\Delta k<<k_{F}$, which is valid in practical device operation. Assuming the electric field and the momentum shift $\Delta k$ are in the $x$ direction, we consider that for each $k_{y}$ the distribution $f\left(k_{x}, k_{y}\right)$ is shifted to $f\left(k_{x}-\Delta k, k_{y}\right)$, and that for each carrier the $x$ direction projection of the velocity is $v_{F} \cdot k_{x} / k$. The drift velocity can therefore be calculated as: 


$$
v_{d}=\frac{4 \int_{0}^{\infty} d k_{y} \int_{0}^{\infty}\left(v_{F} \frac{k_{x}}{k}\right)\left[f\left(k_{x}-\Delta k, k_{y}\right)-f\left(k_{x}, k_{y}\right)\right] d k_{x}}{2 \pi \int_{0}^{\infty} f(k) d k} .
$$

Since $\Delta k$ is small, we have

$$
f\left(k_{x}-\Delta k, k_{y}\right)-f\left(k_{x}, k_{y}\right)=-\frac{\partial f}{\partial k_{x}} \Delta k \text {. }
$$

Inserting (A5) into (A4), we get

$$
v_{d}=\frac{4 v_{F} \int_{0}^{\infty} d k_{y} \int_{0}^{\infty}\left(\frac{k_{x}}{k}\right)\left(-\frac{\partial f}{\partial k_{x}}\right) \Delta k d k_{x}}{2 \pi \int_{0}^{\infty} f(k) d k} .
$$

It can be shown that

$$
v_{d}=v_{F} \frac{\Delta k}{k_{F}} \cdot F_{T}\left(\frac{\hbar v_{F} k_{F}}{k_{B} T}\right) \text {, }
$$

where the temperature factor $F_{T}$ is a function of the ratio $\hbar v_{F} k_{F} /\left(k_{B} T\right)$ and approaches 1 as $\hbar v_{F} k_{F} /\left(k_{B} T\right) \rightarrow \infty$.

For $T>0 \mathrm{~K}$, the carrier density

$$
n=4 \cdot \frac{1}{4 \pi^{2}} \cdot 2 \pi \int_{0}^{\infty} f(k) d k \equiv \frac{k_{F}{ }^{2}}{\pi} \cdot G_{T}\left(\frac{\hbar v_{F} k_{F}}{k_{B} T}\right) .
$$

Again the temperature dependence factor $G_{T}$ depends only on $\hbar v_{F} k_{F} /\left(k_{B} T\right)$ and approaches 1 as ratio $\hbar v_{F} k_{F} /\left(k_{B} T\right) \rightarrow \infty$.

The conductivity is therefore

$$
\sigma=\frac{q^{2} v_{F}}{\hbar \sqrt{\pi}} \cdot \tau \cdot \sqrt{n} \cdot \sqrt{G_{T}\left(\frac{\hbar v_{F} k_{F}}{k_{B} T}\right)} \cdot F_{T}\left(\frac{\hbar v_{F} k_{F}}{k_{B} T}\right) .
$$

The temperature correction factors $G_{T}$ and $F_{T}$ are calculated numerically. Figure A2 shows $\sqrt{n} \cdot \sqrt{G_{T}} \cdot F_{T}$ versus $n$ at $T=300 \mathrm{~K}$, where $k_{F}$ is related to $n$ by Eq. (2). This curve is a scaled version of the $\sigma$ versus $\left|V_{G}-V_{m}\right|$ curve for a monolayer graphene FET. Without fitting to the data in Fig. 2 of Ref. [8], we qualitatively state that the shape of the curve in Fig. A2 agrees with the hole and electron conduction portions of the transfer curve there.

Figure A3 shows $n$ at $T=300 \mathrm{~K}$ versus $\hbar v_{F} k_{F}$ as compared to $k_{F}^{2} / \pi$, as well as the temperature correction factor $\sqrt{G_{T}} \cdot F_{T}$ versus $\hbar v_{F} k_{F}$. The top axis gives $k_{F}$ for reference. For $n=4 \times 10^{12} \mathrm{~cm}^{-2}, \sqrt{G_{T}} \cdot F_{T}=0.98$ and the difference between $n$ and $k_{F}^{2} / \pi$ is negligible, therefore the effect of room temperature is negligible above $n=4 \times 10^{12} \mathrm{~cm}^{-2}$.

The analysis in the Appendix is valid only for Dirac Fermions, i.e. for monolayer graphene or mis-oriented FLG.

\section{Acknowledgement}

This work was supported by DARPA (contract FA8650-08-C-7823), and NSF. Opinions are those of the authors and are not necessarily endorsed by the funding sources. We 
gratefully thank J. Kedzierski and P. Healey of Lincoln Labs for transistor fabrication and testing, TEM, and helpful discussions.

\section{References}

[1] A. K. Geim and K. S. Novoselov, Nature Materials 6 (2007) 183.

[2] K. S. Novoselov, A. K. Geim, S. V. Morozov, D. Jiang, Y. Zhang, S. V. Dubonos, I. V. Grigorieva, and A. A. Firsov, Science 306 (2004) 666.

[3] Th. Seyller, A. Bostwick, K. V. Emtsev, K. Horn, L. Ley, J. L. McChesney, T. Ohta, J. D. Riley, E. Rotenberg, and F. Speck, Phys. Stat. Sol. (b) 245 (2008) 1436.

[4] J. Hass, W. A. de Heer, and E. H. Conrad, J. Phys.: Cond. Mat. 20 (2008) 323202.

[5] G. Gu, S. Nie, R. M. Feenstra, R. P. Devaty, W. J. Choyke, W. K. Chan, and M. G. Kane, Appl. Phys. Lett. 90 (2007) 253507.

[6] Y. Q. Wu, P. D. Ye, M. A. Capano, Y. Xuan, Y. Sui, M. Qi, J. A. Cooper, T. Shen, D. Pandey, G. Prakash, and R. Reifenberger, Appl. Phys. Lett. 92 (2008) 092102.

[7] J. Kedzierski, P.-L. Hsu, P. Healey, P. Wyatt, C. L. Keast, M. Sprinkle, C. Berger, and W. A. de Heer, IEEE Trans. Electron Devices 55 (2008) 2078.

[8] M. C. Lemme, T. J. Echtermeyer, M. Baus, and H. Kurz, IEEE Electron Device Lett. 28 (2007) 282.

[9] Luxmi, S. Nie, P. J. Fisher, and R. M. Feenstra, G. Gu, and Y. Sun, J. Electron. Mater. 38 (2009) 718.

[10] E. H. Hwang, and S. Das Sarma, Phys. Rev. B 75 (2007) 205418.

[11] V. W. Brar, F. Wang, C. Girit, Y. Yayon, M. Panlasigui, A. Zettl, Y. Zhang, and M.

F. Crommie, Nature Phys. 4 (2008) 627.

[12] B. Partoens and F. M. Peeters, Phys. Rev. B 74 (2006) 075404.

[13] A. Grüneis, C. Attaccalite, L. Wirtz, H. Shiozawa, R. Saito, T. Pichler, and A. Rubio, Phys. Rev. B 78 (2008) 205425.

[14] W. A. de Heer, C. Berger, X. Wu, P. N. First, E. H. Conrad, X. Li, T. Li, M.

Sprinkle, J. Hass, M. L. Sadowski, M. Potemski, and G. Martinez, Solid State Comm. 143 (2007) 92.

[15] S. Latil, V. Meunier, and L Henrard, Phys. Rev. B 76 (2007) 201402(R).

[16] J. Hass, F. Varchon, J. E. Millán-Otoya, M. Sprinkle, N. Sharma, W. A. de Heer, C. Berger, P. N. First, L. Magaud, and E. H. Conrad, Phys. Rev. Lett. 100 (2008) 125504.

[17] E. McCann, D. S. L. Abergel, and V. I. Fal'ko, Solid State Comm. 143 (2007) 110.

[18] K. Nomura and A. H. MacDonald, Phys. Rev. Lett. 98 (2007) 076602.

[19] T. Stauber, N. M. R. Peres, and F. Guinea, Phys. Rev. B 76 (2007) 205423.

[20] S. Fratini and F. Guinea, Phys. Rev. B 77 (2008) 195415.

[21] M. I. Katsnelson and K. K. Geim, Phil. Trans. R. Soc. A 366 (2008)195.

[22] J. H. Chen, C. Jang, M. S. Fuhrer, E. D. Williams, and M. Ishigami, Nat. Phys. 4 (2008) 377.

[23] Luxmi, P. J. Fisher, N. Srivastava, and R. M. Feenstra, Y. Sun, J. Kedzierski and P. Healey, and G. Gu, Appl. Phys. Lett. 95 (2009) 073101. 

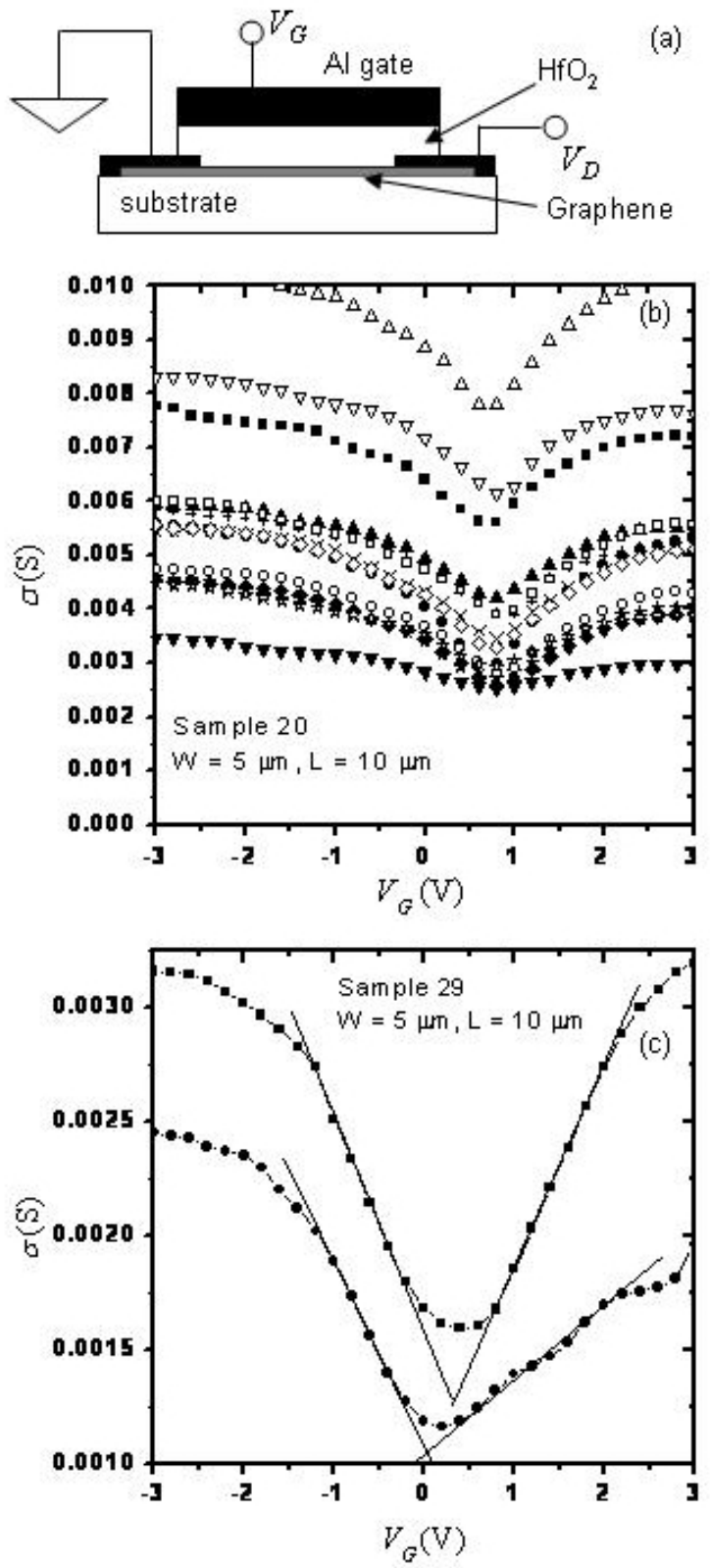

Fig. 1. (a) Device structure. (b) Transfer characteristics (channel conductivity $\sigma$ vs. gate bias $V_{G}$ ) of devices using C-face graphene on sample 20. (c) $\sigma-V_{G}$ curves for two representative devices Si-face graphene on sample 29. The straight lines are visual guidance to emphasize the wide linear ranges of these curves to be discussed later. 


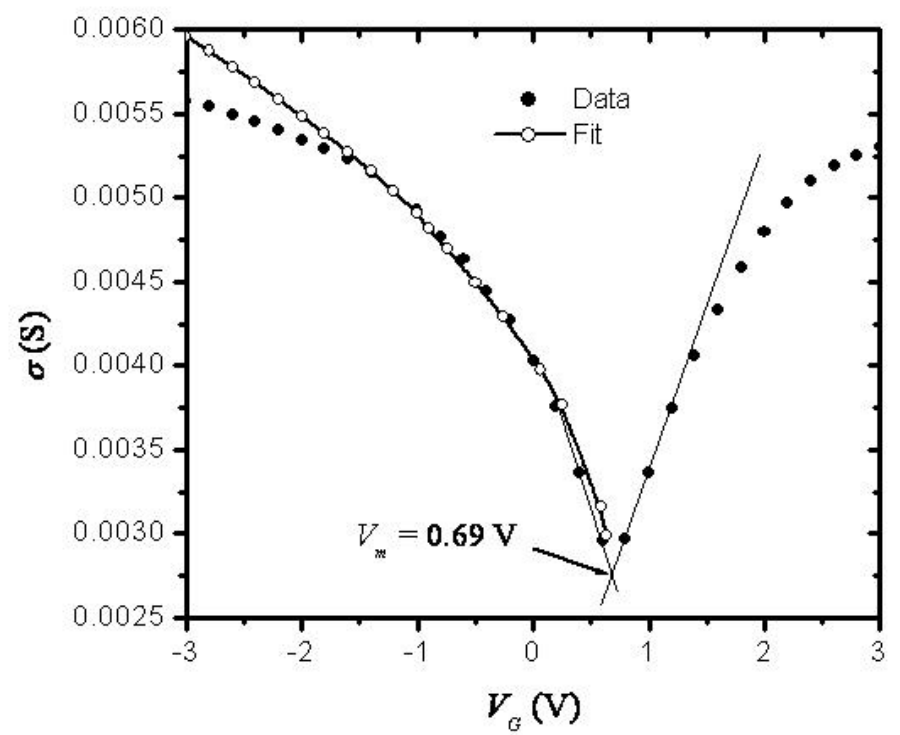

Fig. 2. Channel conductivity vs. gate bias of one C-face graphene FET on sample 20 (Fig. 1(b)). The minimum conductivity point is found by linearly extrapolating the curve near the minimum. The fit matches hole conduction data down close to $V_{m}$, revealing that the C-face graphene has a linear band structure.

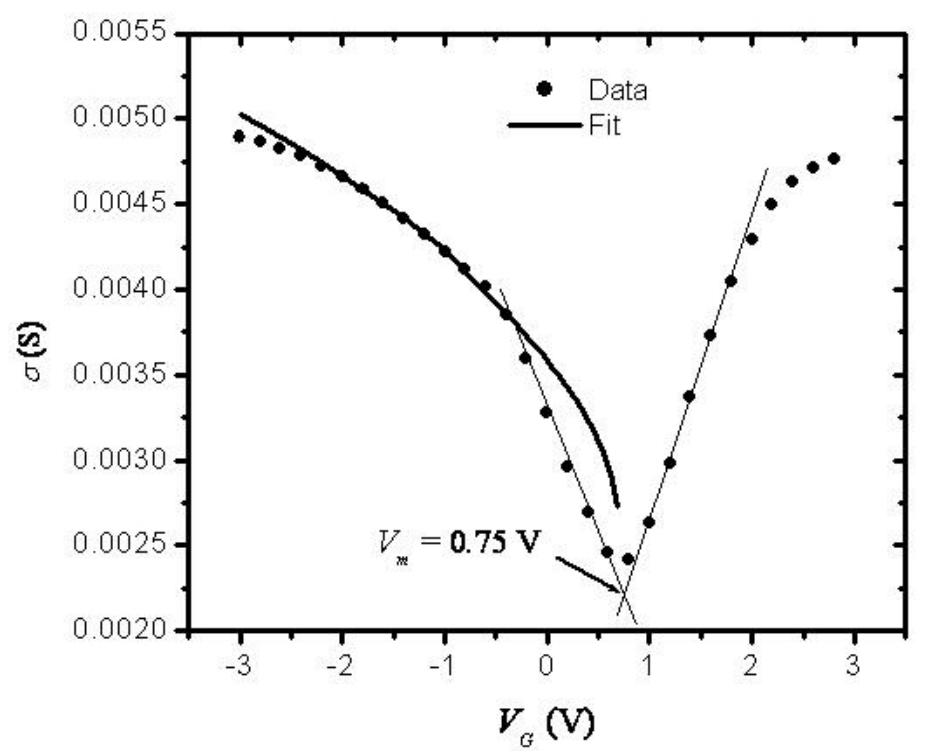

Fig. 3. The transfer characteristics of a Si-face graphene FET on sample 25. The minimum conductivity point is found by linearly extrapolating the curve near the minimum. In contrast to Fig. 2, the fit to hole conduction data is only good down to $V_{G}=$ $-0.6 \mathrm{~V}$, suggesting a different band structure than the linear dispersion. 


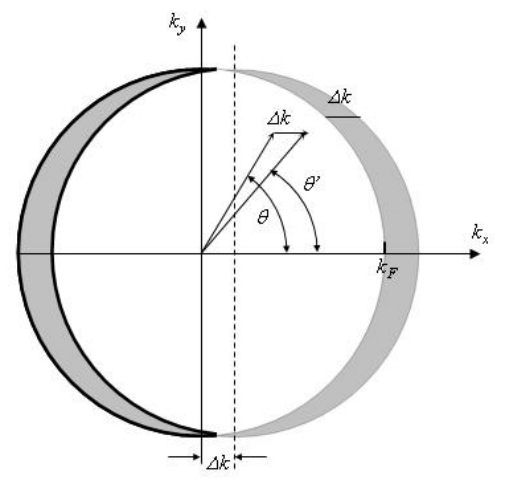

Fig. A1. The momentum is shifted by $\Delta k$ by an applied electric field. When $T=0 \mathrm{~K}$, only the carriers in the shaded areas have net contribution to the drift velocity.

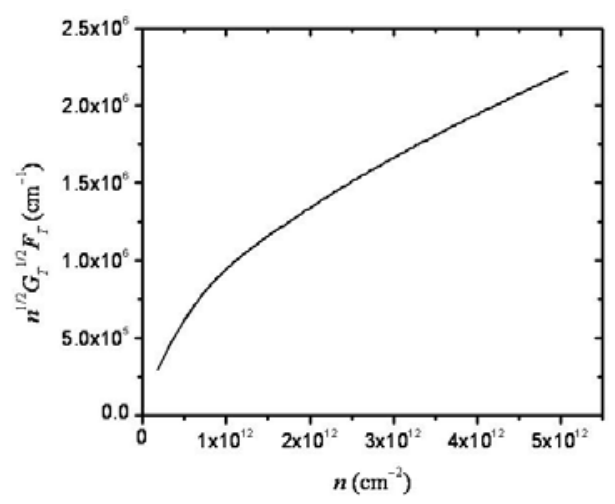

Fig. A2. $\sqrt{n} \cdot \sqrt{G_{T}} \cdot F_{T}$ versus carrier density $n$. Since the channel conductivity is proportional to $\sqrt{n} \cdot \sqrt{G_{T}} \cdot F_{T}$ and $n$ is proportional to the gate overdrive voltage, this curve is a scaled version of the transfer curve of a FET based on graphene with linear energy band dispersion.

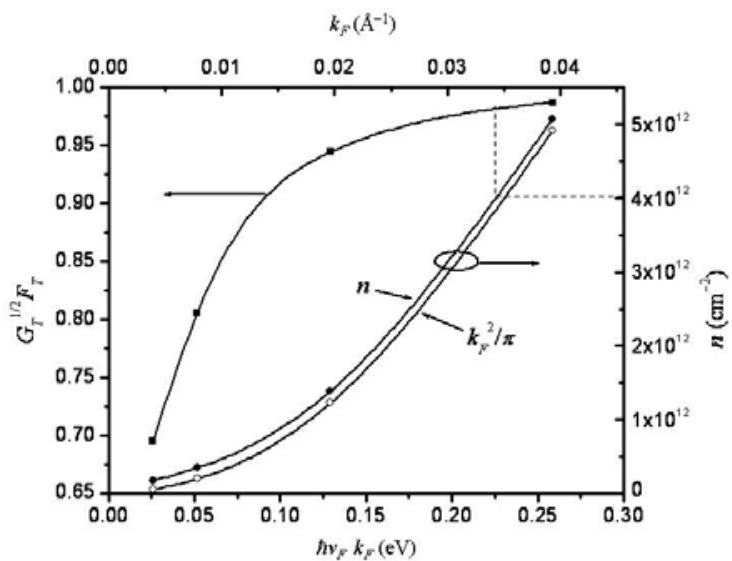

Fig. A3. Carrier density $n$ at $T=300 \mathrm{~K}$ versus $\hbar v_{F} k_{F}$, as compared to $k_{F}^{2} / \pi$ (right axis), and the temperature correction factor $\sqrt{G_{T}} \cdot F_{T}$ (left axis) versus $\hbar v_{F} k_{F}$. The top axis gives $k_{F}$ for reference, assuming $v_{F}=10^{8} \mathrm{~cm} / \mathrm{s}$. For $n=4 \times 10^{12} \mathrm{~cm}^{-2}, \sqrt{G_{T}} \cdot F_{T}=0.98$ and the difference between $n$ and $k_{F}{ }^{2} / \pi$ is negligible, therefore the effect of room temperature is negligible above $n=4 \times 10^{12} \mathrm{~cm}^{-2}$. 